\title{
Utilización de la herramienta Hoja de Cálculo Google en la asignatura Máquinas Eléctricas (Grado en Ingeniería Eléctrica)
}

\section{Paula Bastida-Molina ${ }^{a}$, Carlos Vargas-Salgadob ${ }^{b}$ Lina Montuoric y Manuel Alcázar- Ortega $^{\mathrm{d}}$}

${ }^{a}$ Instituto Universitario de Ingeniería Energética, Camino de Vera s/n, Edificio 8E, $2^{\mathrm{a}}$ planta, Universitat Politècnica de València, paubasmo@etsid.upv.es, bepartamento de Ingeniería Eléctrica. Camino de Vera, s/n Edificio 5E, Universitat Politècnica de València, carvarsa@upvnet.upv.es, ${ }^{\mathrm{c}}$ Departamento de Termodinámica Aplicada, Universitat Politècnica de València, Camino de Vera, s/n, edificio 5J, $2^{\text {a }}$ planta. 46022 Valencia (España), 1montuori@upvnet.upv.es y dDepartamento de Ingeniería Eléctrica. Camino de Vera, s/n Edificio 5E, Universitat Politècnica de València, malcazar@iiie.upv.es

\begin{abstract}
The educational innovation described in this paper has been applied during the $1^{\text {st }}$ practical session of the subject Electrical Machines $\left(2^{\text {nd }}\right.$ course of Electrical Engineering Degree). With this educational innovation, students can introduce the obtained results via on-line using a Google Sheet, since the end of the practical session until a few days before the test. The evaluation is made by this test. In this way, they can observe the results of their colleagues and compare them with their own results, so they are able to detect and correct possible mistakes before the test in an autonomous way by a peer-to-peer comparison. This methodology helps students to also develop abilities like team work or critical thinking. The analysis of the marks obtained this course using Google Sheet tool shows they have substantially improved (the number of passing students has increased up to 28\%). Moreover, the survey answered by the students reflects that their rate of satisfaction is very high too (98\%). This results show that the use and acceptance of this new tool has been satisfactory verified.
\end{abstract}

Keywords: Google Sheet, educational innovation, critical thinking, peer-topeer comparison, team work, computer tool, technological resource.

\section{Resumen}

La innovación educativa descrita en este artículo se ha aplicado en la sesión práctica 1 de la asignatura Máquinas Eléctricas $\left(2^{\circ}\right.$ curso del Grado en Ingeniería Eléctrica). Esta innovación educativa ha consistido en utilizar la herramienta hoja de cálculo Google, para que los estudiantes puedan introducir vía on-line los resultados obtenidos, desde que acaban la práctica hasta unos pocos dias antes del examen test, que es la forma en la que se evalúa la práctica. Pueden visualizar así los resultados de sus compañeros y compañeras y compararlos con los suyos, por lo que son capaces de detectar de forma autónoma y por comparación entre pares si han cometido algún error, pudiendo subsanarlo antes de realizar el test. Esto les permite desarrollar adicionalmente el trabajo en equipo y el pensamiento crítico. El 
análisis de las calificaciones obtenidas este curso al utilizar la hoja de cálculo muestra que estas han mejorado sustancialmente (el número de aprobados/as se ha incrementado en un 28\%). Además, la encuesta realizada al alumnado también muestra que su grado de satisfacción al aplicar esta nueva herramienta es muy alto (98\%). Se comprueba asi que el funcionamiento y la aceptación de esta nueva herramienta ha sido totalmente satisfactorio.

Palabras clave: hoja de cálculo Google, innovación docente, pensamiento crítico, comparación entre pares, trabajo en equipo, herramienta informática, recurso tecnológico.

\section{Introducción}

La educación de los estudiantes no es un proceso estático, sino un proceso dinámico en constante cambio, que debe adaptarse a las nuevas necesidades y herramientas de la sociedad actual (de Miguel Díaz, 2005; Ginés Mora, 2004; Pablos Pons, 2007). Las nuevas tecnologías están transformando la sociedad en la que vivimos y el ámbito de la educación no es una excepción. Es más, en este sector el impacto de la tecnología es cada vez mayor (Baelo Alvarez y Cantón Mayo, 2009; Pablos Pons; Villaciervos Moreno, 2005).

La introducción adecuada de nuevos recursos tecnológicos para la docencia es algo positivo para la misma (Salinas, 2004). Comparando la metodología enseñanza-aprendizaje tradicional con la metodología enseñanza-aprendizaje utilizando nuevas herramientas tecnológicas se ve que uno de los aspectos de esta última en la docencia es que fomenta el trabajo en equipo (trabajo en colaboración, intercambio de información, distintas opciones de comunicación) y centra el aprendizaje en los alumnos y alumnas (aprendizaje enfocado en los alumnos, aprendizaje basado en el pensamiento crítico). En la tabla 1 (Villalobos, 2017) se muestra una comparativa entre el aprendizaje que hoy en día se considera tradicional y el aprendizaje utilizando las nuevas tecnologías

Tabla 1. Comparativa aprendizaje tradicional-aprendizaje nuevas herramientas tecnológicas

\begin{tabular}{cc}
\hline Aprendizaje tradicional & Aprendizaje con nuevas herramientas tecnológicas \\
\hline Trabajo individual & Trabajo en colaboración \\
Comunicación de información lineal & Intercambio de información \\
Único medio de comunicación & Varios medios de comunicación \\
Enseñanza directa del docente & Aprendizaje enfocado en los estudiantes \\
Aprendizaje basado en la experiencia & Aprendizaje basado en el pensamiento crítico \\
\hline
\end{tabular}

Desde la asignatura Máquinas Eléctricas, impartida al alumnado de $2^{\circ}$ curso del Grado en Ingeniería Eléctrica (ETSID-UPV) se ha querido introducir un nuevo recurso tecnológico, para mejorar la calidad de la docencia e intentar mejorar también el proceso de aprendizaje 
de los estudiantes. En concreto, se ha introducido la hoja de cálculo Google durante la sesión práctica 1 de dicha asignatura.

Durante esta sesión práctica, los alumnos y alumnas realizan los cálculos y la simulación de circuitos eléctricos mediante el software Electronics Workbench. Este programa se aplica para determinar los valores de los componentes del circuito equivalente de un transformador. La evaluación de esta práctica se lleva a cabo al inicio de siguiente sesión práctica, mediante un test.

En cursos anteriores, se detectó mediante las calificaciones obtenidas, que a pesar de que el profesor o profesora explica la práctica paso a paso y resuelve las dudas planteadas, había algunos estudiantes que no llegaban a asentar los conocimientos adecuadamente.

Como solución a esta problemática, se ha decidido introducir la hoja de cálculo Google como medida innovadora educativa. Ahora, cuando los alumnos y alumnas finalizan la práctica, pueden introducir los datos obtenidos en la misma en la hoja de cálculo Google, que previamente ha sido configurada y editada por el profesor o profesora. Tienen de tiempo hasta unos pocos días antes del test. De este modo, los estudiantes pueden visualizar los resultados de sus compañeros y compañeras para compararlos con los suyos. Se consigue así que el alumnado pueda darse cuenta de los errores cometidos por si solos realizando una comparación entre pares (Lamas Rojas, 2008), lo que les permite poder subsanarlos antes del test.

\section{Objetivos}

El objetivo principal de este trabajo es utilizar la herramienta informática online hoja de cálculo Google para permitir a los alumnos y alumnas que realizan la práctica 1 de Máquinas Eléctricas ( $2^{\circ}$ curso Grado Ingeniería Eléctrica) analizar los resultados de las pruebas realizadas por comparación entre pares, con el fin de detectar posibles errores y mejorar así el aprovechamiento de la práctica.

De forma paralela al objetivo principal, se han desarrollado una serie de metas adicionales:

- Desarrollo del pensamiento crítico de los alumnos y alumnas ("Pensamiento crítico: Competencias Transversales: UPV," 2019.)

- Trabajo en equipo de los alumnos y alumnas ("Trabajo en equipo: Competencias Transversales: UPV," 2019)

- Utilización de nuevos recursos tecnológicos para la docencia (Lamas Rojas, 2008).

- Recopilación de datos de forma automatizada y sencilla para su posterior tratamiento. 


\section{Desarrollo de la innovación}

\subsection{Descripción de la sesión práctica}

La innovación educativa desarrollada se ha aplicado en la sesión práctica 1 de la asignatura Máquinas Eléctricas, llamada "Simulación de circuitos eléctricos por ordenador", del $2^{\circ}$ curso del Grado en Ingeniería Eléctrica (ETSID- UPV).

Al ser una sesión práctica, el número máximo de estudiantes de cada grupo de prácticas es 25. Para realizarla, los alumnos y alumnas trabajan por parejas, formando subgrupos, cada uno de los cuales dispone de un ordenador.

Mediante esta sesión práctica se pretende que el alumnado conozca cómo calcular el circuito equivalente de un transformador. La práctica está dividida en dos partes, que se describen a continuación:

- En la primera parte, los estudiantes han de calcular y simular una serie de circuitos eléctricos utilizando el software ELECTRONICS WORKBENCH. Además, deben resolver cuestiones relacionadas con dichos circuitos. En esta parte, lo que se pretende es refrescar los conocimientos de cálculo de circuitos eléctricos.

- En la segunda parte de la práctica, los estudiantes simulan el funcionamiento de un transformador a través de su circuito equivalente, utilizando nuevamente el software ELECTRONICS WORKBENCH. Variando la naturaleza de la carga conectada en el secundario del transformador, se puede observar cómo varía la tensión en el secundario y calcular la variación de tensión respecto al funcionamiento en vacío.

Al finalizar la práctica, cada uno de los subgrupos del grupo de prácticas se queda una copia de los resultados obtenidos. Posteriormente, en la siguiente sesión práctica, realizan un examen de tipo test individual para evaluar los conocimientos adquiridos durante la sesión práctica 1 . Este es el método de evaluación de la práctica 1.

\subsection{Problemática detectada en la sesión práctica}

Durante la sesión práctica el profesor o profesora explica en detalle todos los conceptos necesarios para que los alumnos y alumnas puedan desarrollar de forma autónoma el trabajo, resuelve todas las dudas y aclara los problemas planteados. Aun así, de acuerdo con las calificaciones de los tests evaluativos de la práctica 1 de años anteriores, se ha detectado que no todos los estudiantes asimilan completamente los conceptos.

\subsection{Innovación educativa. Hoja de cálculo Google}

El desarrollo de la innovación educativa viene motivado como solución a la problemática detectada en la sesión práctica anteriormente descrita. Consiste en el empleo de una hoja de cálculo Google para recopilar los datos de los alumnos y alumnas. La bibliografía consultada muestra la idoneidad del uso de este tipo de archivos en educación superior (Álvarez Ferrón, Miguel; Sánchez Cañizares, 2014; Marín Díaz, Cabero Almenara y Barroso Osuna, 2015). A continuación, se describe la metodología de la innovación educativa aplicada. 


\subsubsection{Descripción de la Hoja de cálculo Google}

Hoja de cálculo Google es una herramienta informática desarrollada por Google y accesible de forma gratuita para todos los usuarios y usuarias con cuenta de correo electrónico tipo Gmail. Tiene funciones de cálculo muy avanzadas y similares a las hojas de cálculo de Microsoft Excel.

La hoja de cálculo Google es creada por un usuario de Gmail, que será el administrador/a. Este usuario puede compartir la hoja de cálculo con tantos usuarios con cuenta Gmail cómo desee. El administrador es el que gestiona el tipo de actividad que podrán desarrollar el resto de usuarios con los que ha compartido la hoja de cálculo. Por ejemplo, puede darles permiso para editar y visualizar cualquier celda de la hoja de cálculo, para visualizar toda la hoja de cálculo, para sólo modificar ciertas celdas, o para visualizar toda la hoja de cálculo, pero no para editarla etc.

La ventaja principal que presenta esta herramienta es que todos los usuarios y usuarias que tienen acceso a la hoja de cálculo pueden trabajar sobre ella de forma simultánea y de forma on-line.

Se muestra en la figura 1 la hoja de cálculo Google creada por el profesor o profesora para la sesión práctica 1 del grupo 122. En ella se puede ver los distintos parámetros que deben obtener los subgrupos en cada ejercicio de la práctica y que deben introducir en la fila y celda correspondiente, dependiendo del subgrupo en el que estén.

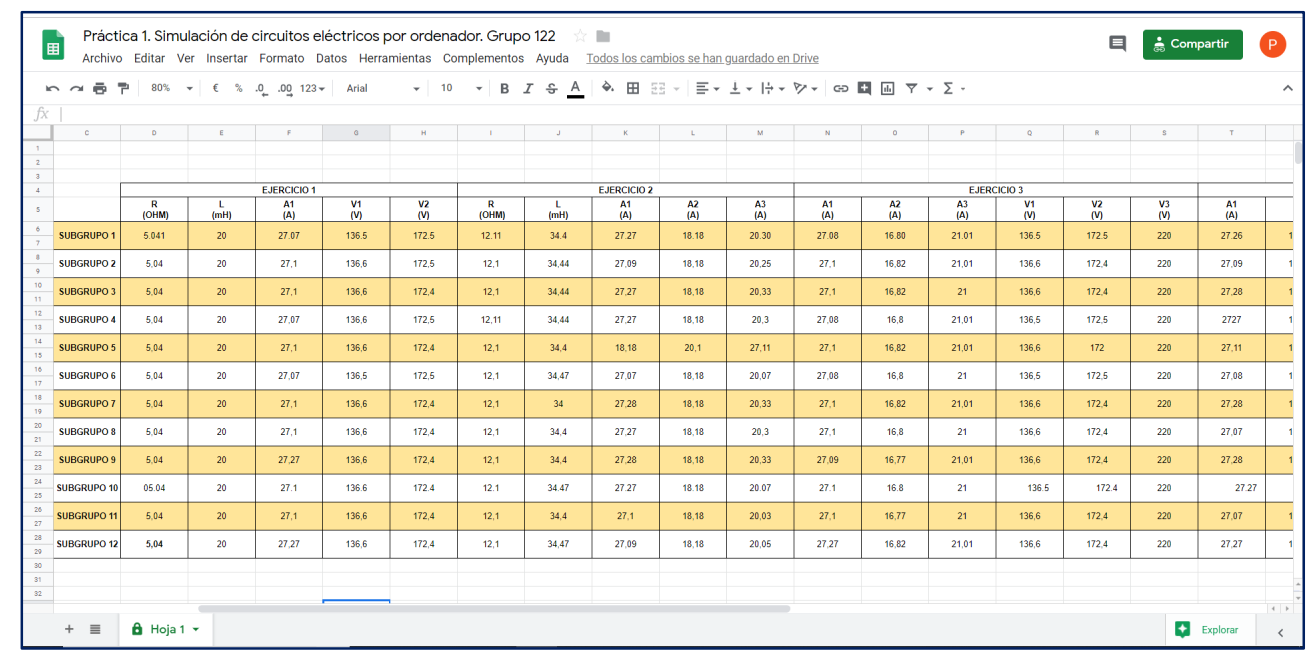

Fig. 1. Hoja de cálculo Google preparada para la sesión práctica 1

2.3.2 Aplicación de la Hoja de cálculo Google en la sesión práctica. Resolución de la problemática detectada en la sesión práctica 1

Aplicar la Hoja de cálculo Google a la sesión práctica "Simulación de circuitos eléctricos por ordenador" ha permitido solucionar el problema que se detectó en esta sesión. Se describe a 
continuación cómo se aplica esta innovación metodológica en la sesión y cómo ha solucionado la problemática existente:

- El profesor o profesora que va a impartir la sesión práctica, crea una hoja de cálculo Google donde los estudiantes tendrán que introducir posteriormente los resultados obtenidos durante la práctica 1 por subgrupos.

- El día de la sesión práctica, el profesor o profesora explica en qué consiste y cómo acceder a la hoja de cálculo Google. También comenta al alumnado que al finalizar la práctica 1 deberán introducir por subgrupos los resultados obtenidos hasta una fecha límite. Pide un correo Gmail a cada subgrupo, para poder darles acceso a la hoja de cálculo Google.

- Para asegurar la comunicación profesor-alumno, el profesor o profesora envía un correo explicando de nuevo a los alumnos y alumnas que deben introducir los resultados obtenidos en la práctica 1 en la hoja de cálculo Google que ha compartido con ellos. Para facilitar el uso de esta nueva tecnología, se les envía también un documento donde se explica cómo acceder a dicha herramienta.

- Los estudiantes pueden ir introduciendo los datos desde que el profesor o profesora les da acceso hasta la fecha límite marcada por el profesor/a. La fecha límite se marca siempre unos días antes del examen tipo test.

- Durante este período de tiempo, cada subgrupo puede introducir sus datos en esta hoja de cálculo Google y a la vez visualizar los datos de sus compañeros y compañeras de forma simultánea y on-line. A través de esta herramienta, lo que se consigue es que los estudiantes sean capaces de discernir de forma autónoma e independiente si los resultados obtenidos durante la sesión práctica fueron correctos por comparación entre pares. Por ejemplo, si el valor de la resistencia del ejercicio 1 introducido por la mayoría de subgrupos está en torno a $5 \Omega$ y el de un subgrupo es de $20 \Omega$, este subgrupo puede detectar de forma autónoma que hay una discrepancia de valores respecto a la de sus compañeros/as.

- Al detectar discrepancias de este tipo, el alumno o alumna es capaz de concebir de forma autónoma que podría tener un error en los resultados ("Pensamiento crítico: Competencias Transversales: UPV," 2019). Como tiene un plazo de tiempo de aproximadamente dos semanas (desde que el profesor o profesora le da acceso a la hoja de cálculo hasta el examen), tiene tiempo para averiguar qué ha ocurrido y tratar de solucionar el error: revisar los cálculos, revisar las simulaciones, repetir la sesión práctica con otro grupo de prácticas, contactar con el profesor/a, etc.

- Al llegar el momento de la evaluación de la práctica 1, el estudiante ha podido asentar mejor los conocimientos de la práctica 1, ya que con la herramienta hoja de cálculo Google ha podido detectar posibles errores. 
El problema presentado en la sesión práctica 1 de Máquinas Eléctricas se ha solucionado mediante esta innovación educativa, desarrollando paralelamente el trabajo en equipo de los estudiantes ("Trabajo en equipo: Competencias Transversales: UPV," 2019), su pensamiento crítico ("Pensamiento crítico: Competencias Transversales: UPV," 2019.), el uso de nuevos recursos tecnológicos y permitiendo además una recopilación de datos de forma automatizada y sencilla para su posible posterior tratamiento.

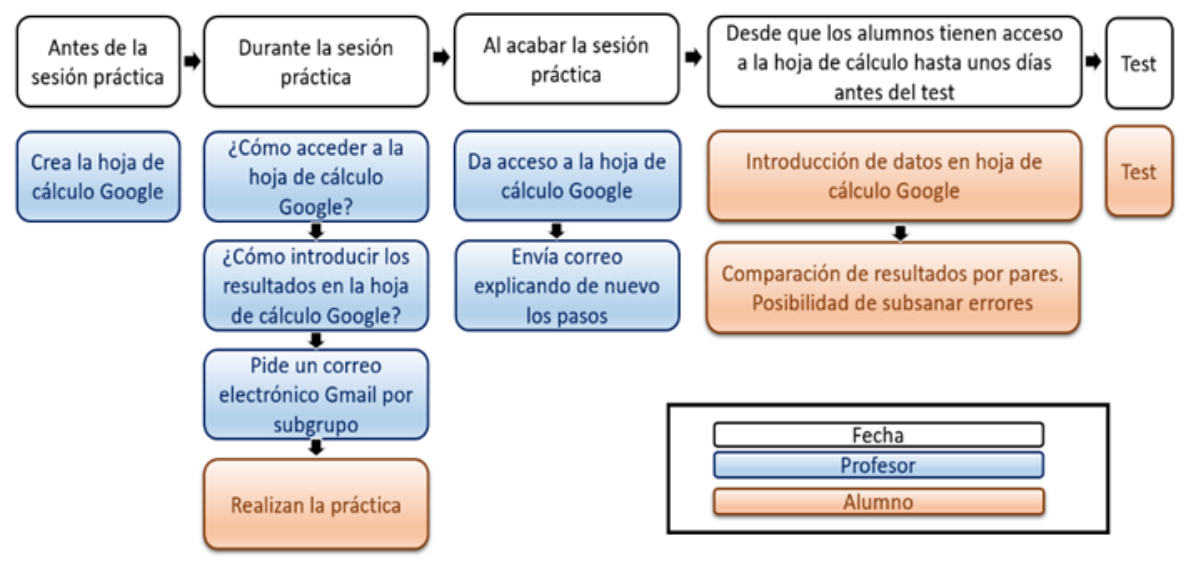

Fig. 2. Aplicación de la hoja de cálculo Google en la sesión práctica 1.

\subsubsection{Posibles problemas que podría introducir la innovación educativa y resolución de los} mismos

La introducción de la innovación educativa en la sesión práctica 1 de Máquinas Eléctricas podría introducir una serie de problemas. Es muy importante detectar esos problemas, para poder darles solución y que la innovación educativa tenga éxito.

A continuación, se describen todos los problemas que podría introducir la innovación educativa y las posibles soluciones de los mismos:

- El problema principal que presentaría la innovación educativa es que sólo permite acceder a la hoja de cálculo Google a través de cuentas Gmail. Los estudiantes de la UPV, por el hecho de ser alumnos y alumnas de dicha universidad, disponen de una cuenta de correo electrónico tipo UPV, pero no tienen por qué tener una cuenta de correo Gmail. Sin embargo, este tipo de cuentas de correo está muy extendido entre la población. Las soluciones que se presentan al problema son las siguientes:

- El alumnado tiene que introducir los resultados por subgrupos. Cada subgrupo está formado por dos estudiantes normalmente, por lo que las probabilidades de que alguno de los miembros tenga cuenta de Gmail son elevadas. 
- Si ninguno de los miembros del subgrupo tuviera cuenta Gmail, la creación de una cuenta de este tipo es muy sencilla y gratuita, por lo que no supondría ningún problema para el estudiante. El profesor o profesora puede ayudarle en esta tarea.

- Otro problema que podría darse al aplicar la innovación educativa es que los estudiantes no supieran cómo acceder a la hoja de cálculo Google compartida por el profesorado. Para solucionar este problema, el profesor o profesora se lo explica durante la sesión práctica y les envía también un correo para asegurar la comunicación profesor-alumno. En este correo, anexa un documento con todos los pasos que deben seguir para acceder a la hoja de cálculo Google.

- Otro problema que podría darse al aplicar la innovación educativa es la inherente a una hoja de cálculo donde diversas personas tienen acceso. Al darles acceso a todos los subgrupos que han realizado la sesión práctica para introducir sus datos, podría darse el caso de que algún subgrupo modificase los resultados introducidos por otro subgrupo. Este problema puede ser solucionado muy fácilmente a través de los permisos de la hoja de cálculo Google. El creador de la hoja de cálculo (en este caso el profesor o profesora) puede restringir el uso de un cierto rango de celdas, según las necesidades: editar y visualizar, sólo visualizar, ni editar ni visualizar...De este modo, en las celdas reservadas para la introducción de datos de cada subgrupo, el profesorado dará permiso para que tan sólo ese subgrupo pueda introducir datos en ese rango de celdas y el resto de grupos sólo pueda visualizar y no editar. Se consigue así proteger el trabajo de los estudiantes, sin desvirtuar el objetivo de la hoja de cálculo Google utilizada en la sesión práctica.

- Además, podrían darse problemas de comunicación, de forma que los estudiantes no reciban la hoja de cálculo Google compartida por el profesor o profesora. En esos casos, el alumno o alumna avisa al profesor o profesora de la incidencia (vía correo electrónico o en persona) y el profesorado revisa la dirección de Gmail con la que ha compartido la página y la vuelve a compartir con el estudiante.

\section{Resultados}

\subsection{Número de estudiantes y subgrupos con cuenta Gmail}

En los cuatro grupos de prácticas de laboratorio $(121,122,123$ y 124) que realizan la sesión práctica 1 se hizo un sondeo para conocer cuántos estudiantes y cuántos subgrupos tenían cuenta de Gmail. Con este sondeo se pretendía conocer lo estandarizado que estaba el uso de las cuentas Gmail entre los mismos. 
El resultado fue muy satisfactorio. En total, 78 alumnos y alumnas de 81 tenían cuenta Gmail (78\%) y todos los subgrupos disponían de cuenta Gmail (100\%), lo que demuestra que el uso de las cuentas Gmail está muy estandarizo entre el alumnado.

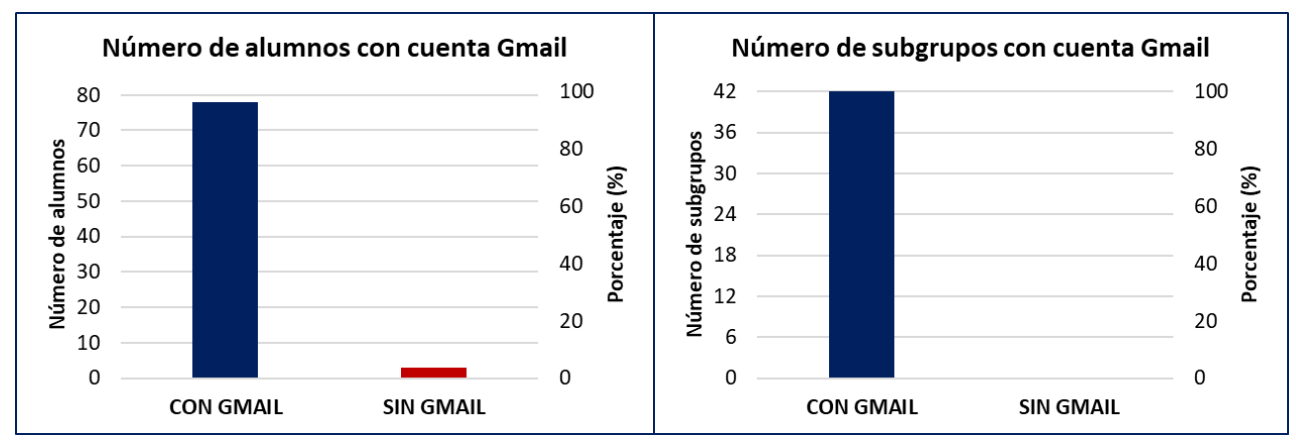

Fig. 3. Número de alumnos y alumnas y subgrupos con cuenta Gmail

\subsection{Mejora de los resultados en la sesión práctica}

El objetivo principal que se ha querido alcanzar al aplicar esta innovación educativa ha sido utilizar una herramienta informática online que permitiese a todos los estudiantes del grupo escribir sus propios resultados y visualizar los de sus compañeros y compañeras, con el fin de detectar posibles errores por comparación entre pares (Lamas Rojas, 2008) y mejorar así el aprovechamiento de la docencia.

La forma cuantitativa de comprobar el impacto de la innovación educativa ha sido a través de los resultados de los test evaluativos de la sesión práctica 1 que realiza el alumnado.

Se ha detectado que ha habido una mejora significativa de los resultados respecto de los resultados obtenidos en años anteriores. Como se puede ver en la figura 4, el número de aprobados y aprobadas total respecto el año pasado, cuando no se utilizaba la hoja de cálculo Google, ha aumentado un $28 \%$, ya que en 2018 el porcentaje de aprobados y aprobadas fue de un $67 \%$, aumentando este año hasta un $86 \%$. Además, si se comparan las calificaciones por franjas numéricas, se observa que las notas por debajo de 2,5 han descendido desde el $12 \%$ en 2018 hasta un $6 \%$ en 2019 (decremento del 47\%) y las notas entre 2,5 y 5 desde un $21 \%$ en 2018 hasta un $7.5 \%$ en 2019 (decremento del 65\%). Por otro lado, las calificaciones entre 5 y 7.5 han aumentado desde un 49\% en 2018 hasta un 62\% en 2019 (aumento del 26\%) y las calificaciones superiores a 7.5 han pasado de $19 \%$ en 2018 a un $25 \%$ en 2019 (aumento del $33 \%)$. 
Estos resultados son un indicador numérico de la mejora que ha supuesto el uso de la hoja de cálculo Google para los estudiantes.

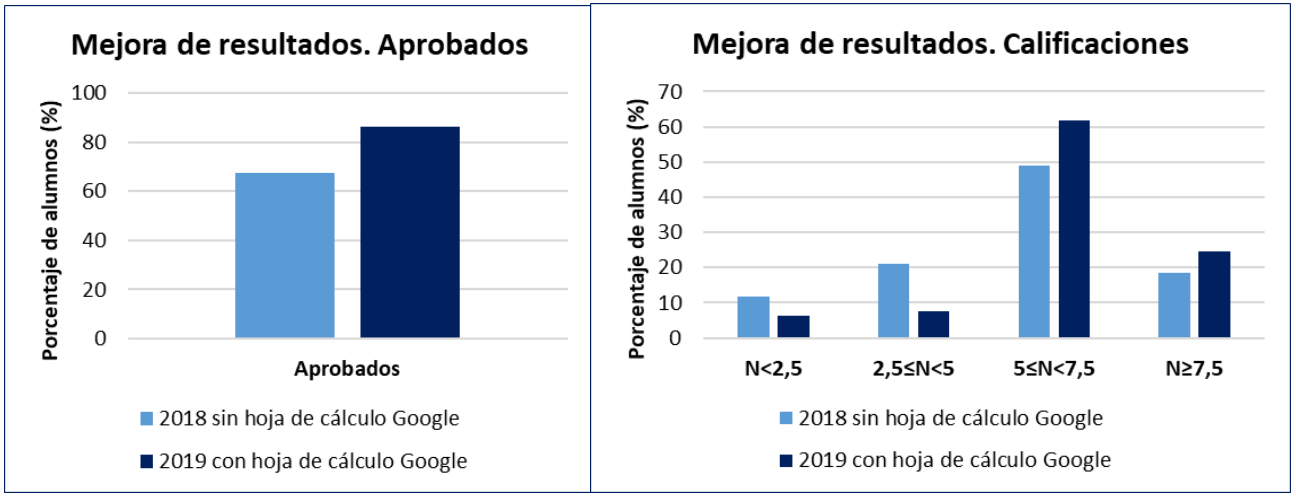

Fig. 4. Mejora de resultados. Aprobados y aprobadas. Calificaciones.

De forma cualitativa, el profesorado de la asignatura ha percibido también una mayor implicación de los estudiantes por afianzar los conocimientos adquiridos durante la práctica 1 .

Se han recibido 27 correos electrónicos referidos a cuestiones relacionadas con la práctica 1 y se han solicitado e impartido 4 tutorías individuales. A través de las cuestiones planteadas tanto en las tutorías como en los correos electrónicos se ha notado el interés del alumnado por afianzar sus conocimientos. Teniendo en cuenta que en total son 81 alumnos y alumnas, se considera que la participación de los mismos ha sido muy alta. Además, comparando las consultas y tutorías atendidas con las de otros años ( 5 y 1 , respectivamente), se percibe una mejora muy importante.

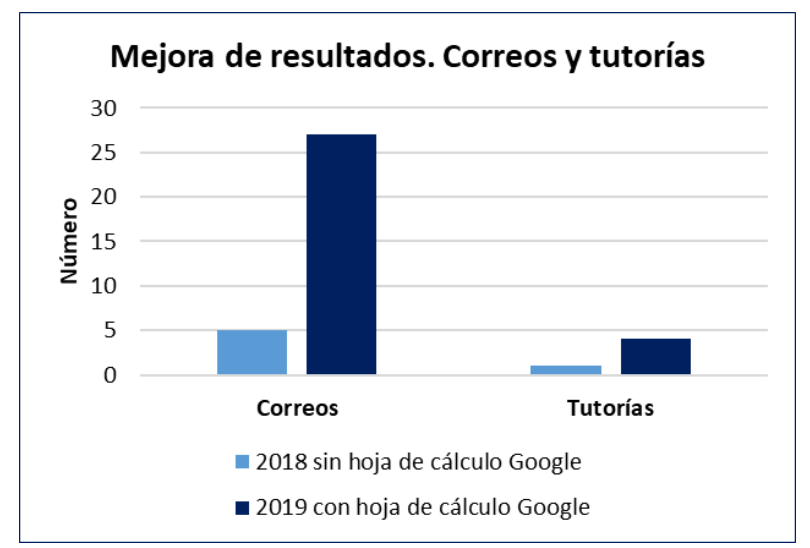

Fig. 5. Mejora de resultados. Correos y tutorías.

\subsection{Opinión de los estudiantes}

Las calificaciones de los test evaluativos y la motivación percibida por el profesorado de la sesión práctica 1 han mejorado sustancialmente respecto a las de otros años, tal y como se ha 
comentado. Aun así, se cree que es muy importante conocer la opinión de los estudiantes en cualquier aspecto de la docencia, especialmente cuando hay cambios o se introducen mejoras como la explicada en este artículo (Harden, RM; Crosby, 2000).

Aprovechando que todos los subgrupos tenían cuenta de Gmail, como se comprobó en el sondeo que se hizo, se creó un formulario Google para conocer la opinión de los estudiantes respecto a la introducción de la hoja de cálculo Google en la sesión práctica 1.

Formulario Google es otra herramienta disponible y gratuita para los usuarios y usuarias con cuenta Gmail, cada vez más utilizada para realizar encuestas on-line (Jhonnel Alarco, J ; Álvarez-Andrade, 2012). El administrador o administradora, que en este caso es el profesor o profesora, diseña un cuestionario y a continuación lo comparte con los usuarios y usuarias con cuenta Gmail que desee, que en este caso eran los estudiantes que habían realizado la sesión práctica 1 de Máquinas Eléctricas.

Como el trabajo se ha realizado por subgrupos, se ha decidido enviar este formulario a cada subgrupo.

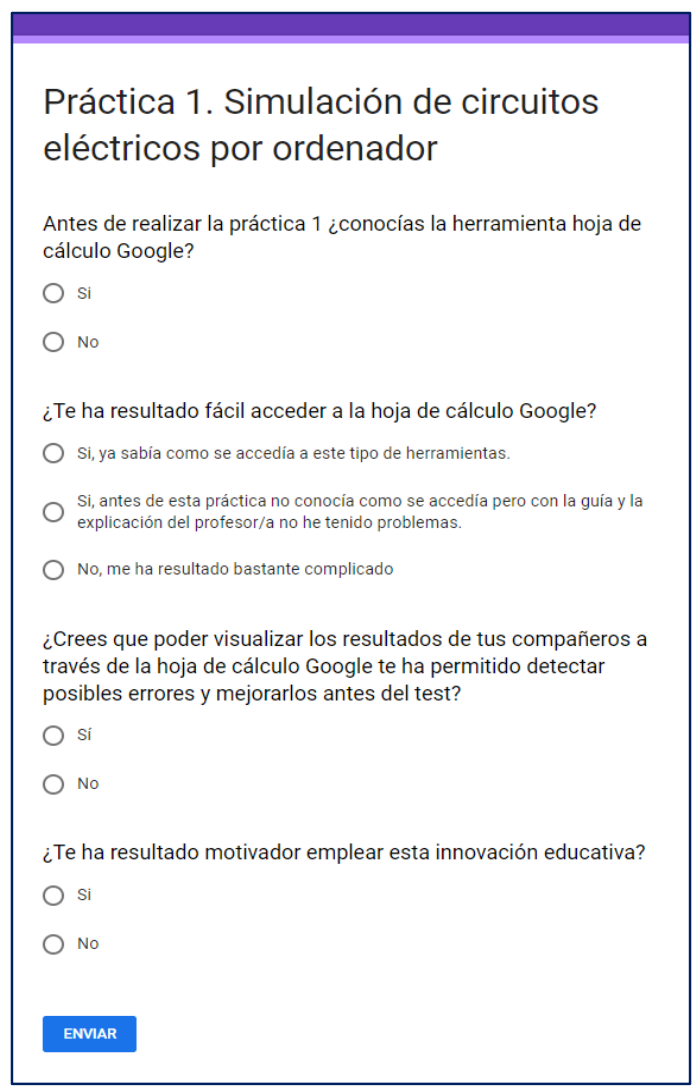

Fig. 6. Opinión de los estudiantes. Encuesta Formulario Google

Los resultados obtenidos a partir de este formulario han mostrado que los estudiantes, en general, consideran que la introducción de la innovación educativa en cuestión ha sido un aspecto muy positivo. A pesar de que muy pocos subgrupos conocían la herramienta hoja de cálculo Google antes de la sesión práctica (sólo un 12\%), a la mayoría le ha resultado fácil 
acceder a la misma con las explicaciones del profesor/a y con la guía de ayuda que se les proporciona (86\%). Una amplísima mayoría de los subgrupos considera también que haber podido visualizar los resultados de sus compañeros y compañeras a través de la hoja de cálculo Google le ha permitido detectar posibles errores y mejorarlos antes del test (98\%). Por último, el $69 \%$ del alumnado opina que utilizar esta innovación educativa ha resultado motivador para ellos.
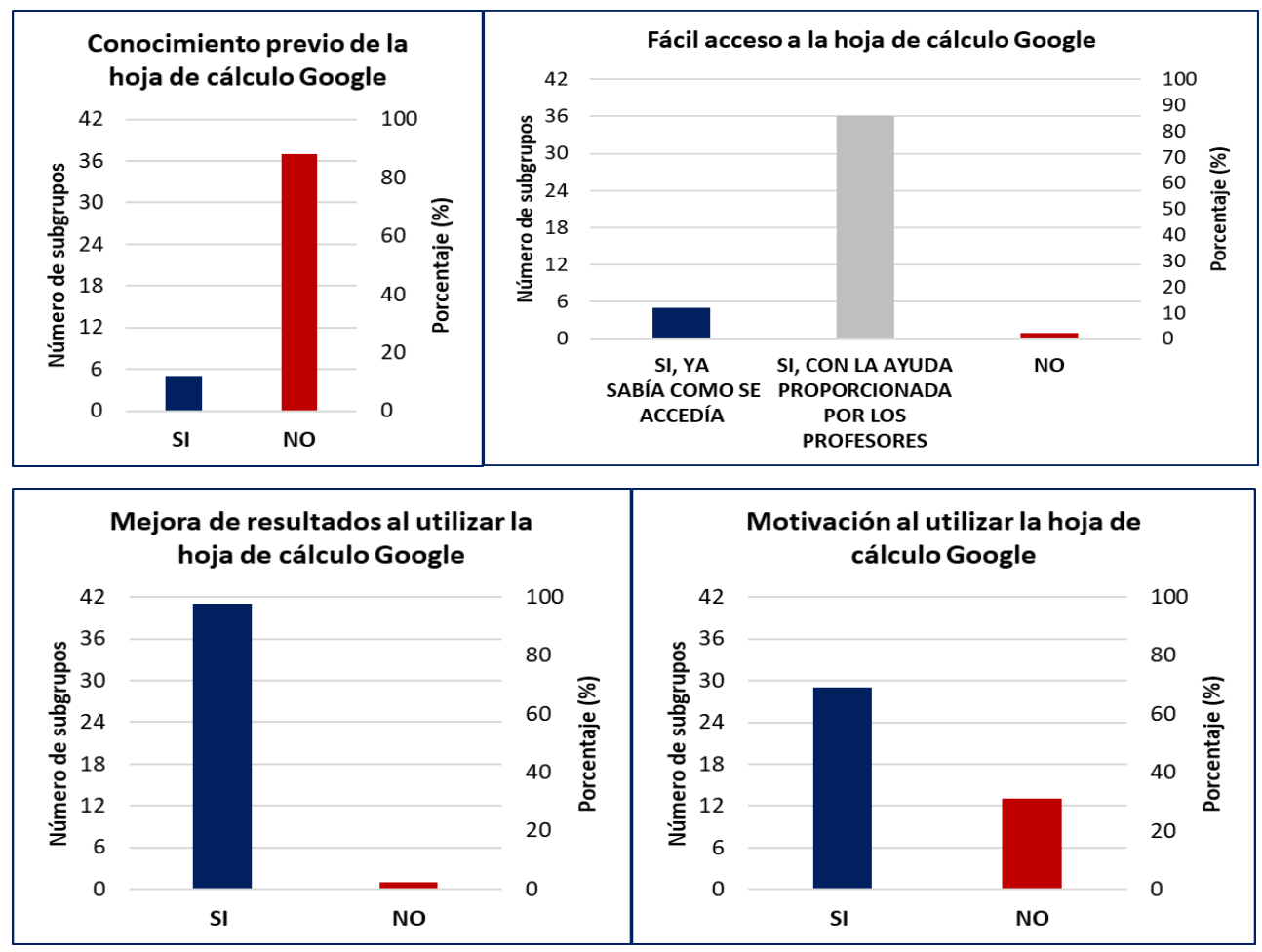

Fig. 7. Opinión de los estudiantes.

\section{Conclusiones}

Esta comunicación presenta la introducción de la hoja de cálculo Google durante la sesión práctica 1 de la asignatura Máquinas Eléctricas, de $2^{\circ}$ curso del Grado en Ingeniería Eléctrica. Tal y como se describe, esta medida innovadora educativa se introdujo para solucionar la problemática detectada en dicha sesión: los estudiantes no llegaban a asentar los conocimientos de la práctica 1 , tal y como los resultados de los test evaluativos advirtieron al profesorado.

Después de la explicación del uso de esta nueva herramienta y su aplicación a la sesión práctica 1 de Máquinas Eléctricas, se muestran los resultados de los análisis cuantitativos y cualitativos realizados. En el análisis cuantitativo se han comparado las calificaciones obtenidas por los estudiantes del año pasado, cuando aún no usaban la hoja de cálculo Google, con las de este año, en el que cual los estudiantes ya han utilizado dicha herramienta. 
Se ha observado una mejora significativa de las calificaciones: el número de aprobados y aprobadas se ha incrementado en un $28 \%$, las notas por debajo de 2.5 se han reducido en un $47 \%$, las notas entre 2.5 y 5 también se han reducido en $65 \%$; por otro lado, las calificaciones entre 5 y 7.5 han aumentado un $26 \%$ y las notas superiores a 7.5 se han incrementado en un $33 \%$. De forma cualitativa, se ha comprobado que el interés del alumnado en afianzar los conocimientos de la sesión práctica 1 es muy alto. Esto se ha percibido a través de las consultas y tutorías solicitadas.

Finalmente, se ha querido valorar también el grado de satisfacción de los estudiantes al introducir esta innovación educativa a través de una encuesta. Los resultados de la misma muestran que a pesar de que muy pocos subgrupos conocían la herramienta antes de la práctica (sólo un $12 \%$ ), a la mayoría le ha resultado fácil acceder a la misma con las explicaciones del profesor/a y con la guía de ayuda que se les proporciona (86\%). Una amplísima mayoría de los subgrupos considera también que haber podido visualizar los resultados de sus compañeros y compañeras a través de la hoja de cálculo Google le ha permitido detectar posibles errores y mejorarlos antes del test (98\%). Por último, el 69\% del alumnado opina que utilizar esta innovación educativa ha resultado motivador para ellos.

Los resultados recopilados demuestran así que la introducción de la hoja de cálculo Google como innovación educativa aplicada en la sesión práctica 1 de Máquinas Eléctricas ha permitido mejorar el asentamiento de los conocimientos de esta práctica en los alumnos y alumnas, desarrollando paralelamente su pensamiento crítico y el trabajo en equipo. Además, la hoja de cálculo Google es un nuevo recurso tecnológico para la docencia que permite recopilar los datos de forma automatizada para su posterior tratamiento.

\section{Agradecimientos}

Este trabajo ha sido respaldado en parte por la administración pública de Valencia bajo la beca ACIF/2018/106.

\section{Referencias}

ÁLVAREZ FERRÓN, M y SÁNCHEZ CAÑIZARES, L. (2014). "Conocimiento, valoración y utilización por parte del alumnado, de Google Drive como herramienta de trabajo cooperativo". Enseñanza \& Teaching: Revista Interuniversitaria de Didáctica, vol. 32, issue 2, p. 23-52.

BAELO ALVAREZ, R y CANTÓN MAYO, I. (2009). "Las tecnologías de la información y la comunicación en la educación superior. Estudio descriptivo y de revisión". Revista Iberoamericana de Educación, vol. 50, issue 7, p. 1-12.

DE MIGUEL DÍAZ, M. (2005). "Cambio de paradigma metodológico en la Educación Superior Exigencias que conlleva". Cuadernos de Integración Europea, p.16-27.

GINÉS MORA, J. (2004). "La necesidad del cambio educativo para la sociedad del conocimiento". Revista Iberoamericana de La Educación, vol. 35, p. 13-37

HARDEN, RM y CROSBY, J. (2000). "The good teacher is more than a lecturer - the twelve 
roles of the teacher". Medical Teacher, vol. 22, p. 334-347.

JHONNEL ALARCO, Jy ÁLVAREZ-ANDRADE, V. (2012). "Google Docs: una alternativa de encuestas online". Educ Med, vol. 15, issue 1, p. 9-10.

LAMAS ROJAS, H. (2008). "Aprendizaje autorregulado, motivación y rendimiento académico". Liberabit, vol. 14, issue 14, p. 15-20.

MARÍN DÍAZ, V, CABERO ALMENARA, J y BARROSO OSUNA, J. M. (2015). "Promoviendo el Uso de Google Drive como Herramienta de Trabajo Colaborativo en la Nube para Estudiantes de Ingeniería". Eduweb, vol. 8, issue. 1,p. 43-56

PABLOS PONS, J; VILLACIERVOS MORENO, P. (2005). "El espacio Europeo de Educación Superior y las tecnologías de la información y la comunicación. Percepciones y demandas del profesorado". Revista de Educación, vol. 337, p. 99-124.

PABLOS PONS, J (2007). "El cambio metodológico en el Espacio Europeo de Educación Superior y el papel de las tecnologías de la información y la comunicación". RIED: Revista Iberoamericana de Educación a Distancia, vol. 10, issue. 2, p. 15-44

SALINAS, J. (2004). "Innovación docente y uso de las TIC en la enseñanza universitaria". RUSC. Revista Universidad y Sociedad Del Conocimiento, vol. 1, p. 1-16.

UNIVERSITAT POLITÈCNICA DE VALÈNCIA. Pensamiento crítico: Competencias Transversales: UPV.

$<$ http://www.upv.es/contenidos/COMPTRAN/info/955136normalc.html> [Consulta: 15 de marzo de 2019]

UNIVERSITAT POLITÈCNICA DE VALÈNCIA. Trabajo en equipo: Competencias Transversales: UPV.

$<$ http://www.upv.es/contenidos/COMPTRAN/info/954872normalc.html> [Consulta: 15 de marzo de 2019]

VILLALOBOS GORDILLO, MC. Herramientas Tecnológicas en la Educación. $<$ https://www.researchgate.net/publication/321070484_Herramientas_Tecnologicas_e n_la_Educacion> [Consulta: 14 de marzo de 2019] 